\title{
Los programas de inclusión social juvenil en la gestión de las violencias de género: reflexiones a partir de la implementación del Programa Envión en Buenos Aires
}

DOI: https://doi.org/10.18046/recs.i35.4658

\author{
Youth Social Inclusion Programs in the Management of Gender \\ Violence: Reflections from the Implementation of the Envion \\ Program in Buenos Aires
}

\author{
Ana Cecilia Gaitán ${ }^{* *}$ \\ Universidad Nacional de San Martín (Buenos Aires, Argentina)
}

\footnotetext{
* Este artículo se deriva del proyecto de investigación "Mujeres, autonomía y participación. Un análisis sobre las continuidades y discontinuidades en las propuestas estatales para la inclusión social de jóvenes de sectores populares en Buenos Aires", financiado por el CONICET (Argentina) a través de una beca posdoctoral (2018-2020). También contó con el apoyo de la Secretaría de Relaciones Exteriores mexicana (SRE) a través de la Beca de Excelencia para extranjeros del Gobierno de México (2019). Artículo de investigación recibido el 17.02.2021 y aceptado el 08.07.2021.

** Doctora en Antropología de la Universidad de Buenos Aires (UBA, Argentina). Investigadora asistente del Consejo Nacional de Investigaciones Científicas y Técnicas de Argentina (CONICET) con sede de trabajo en el Laboratorio de Investigaciones en Ciencias Humanas (LICH) de la Universidad Nacional de San Martín (UNSAM, Argentina). Docente en la UBA y en la UNSAM. Integrante del Programa de Estudios Sociales en Género, Infancia y Juventud (CEDESI-UNSAM). Correo electrónico: agaitan@unsam.edu.ar ORCID: https://orcid.org/oooo-ooo2-7972-9034
} 


\section{Cómo citar/How to cite}

Gaitán, Ana Cecilia (2021). Los programas de inclusión social juvenil en la gestión de las violencias de género: reflexiones a partir de la implementación del Programa Envión en Buenos Aires. Revista CS, 35, 99-123. https://doi.org/10.18046/recs.i35.4658 


\section{Resumen}

En las dos últimas décadas, tanto en Argentina como en el ámbito regional, ha emergido un conjunto de programas destinados a jóvenes con derechos vulnerados o en riesgo de exclusión, tendientes a garantizar su integración al sistema educativo y al mercado laboral. A partir del análisis del programa Envión en la provincia de Buenos Aires, este artículo ofrece reflexiones sobre la manera en que estas iniciativas de inclusión social pueden también operar como dispositivos encargados de gestionar el sufrimiento de mujeres jóvenes pobres; y sobre el modo en que dicha gestión puede presentar ambivalencias y tensiones entre destinatarias y trabajadoras que invitan a explorar con detenimiento las relaciones entre jóvenes pobres, violencias y cuidados. Los datos presentados fueron producidos en el marco de dos investigaciones cualitativas centradas en los procesos participativos orquestados por el Envión entre 2013 y 2017.

\section{PALABRAS CLAVE:}

Estado, mujeres jóvenes, sufrimiento social, violencias de género

In the last two decades in Argentina and at the regional level, a set of programs aimed at young people with violated rights or at risk of exclusion have emerged, aimed at guaranteeing their integration into the education system and the labor market. Based on the analysis of the Envión Program in the province of Buenos Aires, this article offers reflections on how these social inclusion initiatives can also operate as devices for managing the suffering of poor young women, and on how such management can present ambivalences and tensions between clients and state workers, which invite a careful exploration of the relationships between poor young people, violence, and care. The data presented was produced within the framework of two qualitative investigations focused on the participatory processes orchestrated by Envión between 2013 and 2017.

\section{KEYWORDS:}

State, Young Women, Social Suffering, Gender Violence 



\section{Introducción}

En el ámbito regional, durante las dos últimas décadas ha emergido un conjunto de programas destinados a adolescentes y jóvenes con derechos vulnerados o en riesgo de exclusión, tendientes a garantizar su integración en el sistema educativo y al mercado laboral. En Argentina, el surgimiento de estos programas se enmarcó en el contexto de institucionalización de los derechos de niñes y adolescentes y se vinculó a la pretensión estatal de refundar su vínculo con la juventud pobre (Llobet et al., 2013). Como lo indiqué en un artículo previo (Gaitán, 2019), y de acuerdo con los señalamientos expuestos en Llobet, Gaitán, Medan y Magistris (2013), estos programas propusieron metodologías de trabajo más flexibles; en ellos, pretensiones de horizontalidad y conceptos de la educación popular se combinaban con la idea de voluntariedad, plasmada en un contrato ${ }^{1}$ a través del cual les destinataries se comprometían con su situación (Gaitán, 2019:289). Además, para conseguir aquella distancia respecto de actores estatales represivos -policía o poder judicial- y tradicionales -escuela- y lograr acercarse de un modo diferente a les jóvenes, apelaban a la acción y saberes de militantes sociales y referentes territoriales, y establecían los barrios donde aquelles vivían como espacios estratégicos para la intervención (Llobet et al., 2013, como se citó en Gaitán, 2019: 289).

Estos programas han sido objeto de análisis de investigaciones locales que han avanzado en indicar al menos dos cuestiones significativas sobre ellos. En primer lugar, la manera paradójica en que generan disciplinamiento, a la vez que habilitan la ampliación de ciudadanía, bien porque favorecen el acceso directo a derechos o porque crean condiciones para que les jóvenes aprendan a demandarlos (Llobet $e t$ al., 2013). En segundo lugar, en la construcción del riesgo y de la exclusión inherente a sus definiciones institucionales, estos programas visibilizan algunas situaciones como riesgosas y problemáticas, mientras invisibilizan otras (Gaitán, 2017; Llobet, 2008; Medan, 2013).

Con la recuperación de dichos aportes, en el presente artículo busco ofrecer nuevas reflexiones respecto a la manera en que aquellos programas estatales pueden ser leídos como dispositivos encargados de gestionar el sufrimiento de mujeres jóvenes pobres. Esto en tanto, de un lado, reconocen la condición de vulnerabilidad de estas

1. Utilizo itálicas para introducir la voz nativa y términos propios de las sujetas de investigación. Estas introducciones se producen a través de pasajes de entrevistas y registros de campo. También las utilizo para relativizar determinados términos o indicar su polisemia. Además, cabe señalar que opto por utilizar el lenguaje inclusivo a partir del uso de la letra $e$, con dos propósitos: expresar la diversidad y complejidad de las relaciones sociales e identidades, y cuestionar el binarismo con el que han sido pensadas. En cuanto a las destinatarias y trabajadoras del programa, interlocutoras directas de ambas investigaciones, me refiero a ellas como mujeres, en tanto se autopercibían como mujeres cisgénero. 
mujeres, relativizada por otros actores sociales como, por ejemplo, los medios de comunicación masivos que (re)producen estereotipos de aquellas como malas victimas de las violencias de género (Arduino, 2014); y de otro lado, despliegan acciones de protección en esa línea (Llobet, 2015). Para ello me centraré en Envión: este es uno de los programas de la provincia de Buenos Aires dirigidos a la población juvenil con mayor alcance. Indicaré la forma en que este programa, destinado a jóvenes de sectores populares, constituía un escenario particular de un proceso social más amplio de gestión de las vulnerabilidades y violencias de género.

Los datos que presento fueron producidos en el marco de dos investigaciones que han puesto el foco en los procesos participativos (Cornwall, 2008), orquestados por el programa de inclusión social juvenil Envión, en su encarnadura en dispositivos juveniles locales en dos municipios del conurbano bonaerense entre los años 2012 y 2017. En consonancia con el planteamiento de Andrea Cornwall (2008), considero las propuestas de participación orquestadas por este programa como procesos políticos en que discursos institucionales, traducidos en prácticas concretas, se despliegan para regular las acciones de las destinatarias dentro y fuera del programa. En estos procesos participativos, las jóvenes mujeres pueden transgredir sus posiciones como destinatarias pasivas y refutar los intentos de gobierno que recaen sobre ellas.

El artículo se encuentra organizado en cuatro apartados. En el primero repongo aspectos metodológicos centrales de ambas investigaciones; allí detallo las estrategias priorizadas para la construcción y análisis de datos. En el segundo presento aspectos generales del programa Envión. En el tercero aporto luces sobre los trabajos reflexivos puestos en juego en dos propuestas participativas elaboradas localmente para gestionar las violencias que enfrentaban las destinatarias; atiendo tanto el trabajo reflexivo de las agentes estatales e investigadoras encargadas de diseñar y llevar adelante dichas propuestas, como el de las destinatarias. En las conclusiones señalo cómo aquellas propuestas de participación que Envión ofrecía a las jóvenes mujeres, y que se erigían y tramaban sobre una labor reflexiva tendiente a redefinir sus identidades, lejos de ser armoniosas, presentaban ambivalencias y tensiones que invitan a explorar con detenimiento las relaciones que las mujeres jóvenes pobres mantienen con las violencias y los cuidados-invitación que fuera también colocada recientemente por otras investigaciones en Argentina- (Cozzi, 2019; Masson, 2020).

\section{Sobre la producción y análisis de los datos}

Como lo mencioné en el apartado introductorio, los datos presentados aquí fueron producidos en el marco de dos investigaciones que se centraron en los procesos participativos (Cornwall, 2008) orquestados por el programa Envión. La primera, 
de carácter etnográfico, corresponde a mi pesquisa doctoral (2012-2016): en ella procuré aportar a la comprensión de los modos de gestión contemporáneos de la maternidad juvenil y el género en el marco de programas estatales destinados a la inclusión social de jóvenes. El trabajo de campo fue realizado principalmente en la Casa de la Juventud La Estrella ${ }^{2}$, una de las sedes del Envión en el municipio de Morón. Dado que el eje central de la perspectiva etnográfica es el análisis de las situaciones que se viven cotidianamente y suelen no ser documentadas (Achilli, 2005), optar por aquella perspectiva permitió brindar claridades sobre aquello que refería a lo informal e intersticial de la política social considerada, así como captar la complejidad y no linealidad de las relaciones sociales que se producían cotidianamente en el marco de su implementación (Cerletti; Gessaghi, 2012). En esta investigación empleé diferentes estrategias metodológicas. Si bien llevé a cabo entrevistas semiestructuradas, abiertas y en profundidad, y relevé y analicé fuentes secundarias, privilegié la "participación con observación” (Guber, 2004). ${ }^{3}$

La segunda pesquisa, en cambio, se trató de una investigación - acción participativa (Sirvent; Rigal, 2014) realizada en el año 2016. Esta se inscribe en una historia de trabajo conjunto entre el Programa de Estudios Sociales en Género, Infancia y Juventud de la Universidad Nacional de San Martín y el Centro Juvenil la Llamita, uno de los lugares donde se implementaba el programa Envión en el municipio de San Martín. Este segundo proyecto emergió tras la demanda de dicho Centro de que el Programa de Estudios Sociales en Género, Infancia y Juventud, espacio en el que me desempeño como investigadora, colaborase en el fortalecimiento de la participación de sus jóvenes destinatarias ${ }^{4}$. Para lograr este objetivo, el Centro Juvenil nos propuso diseñar un espacio propio para ellas. En la planificación conjunta entre el Centro y el Programa, aquel espacio se pensó con una dinámica flexible que permitiese recoger los emergentes de cada encuentro e integrarlos al objetivo de la iniciativa: habilitar un ambiente seguro y de confianza en el que las destinatarias pudiesen construir vínculos con otras mujeres, a partir de problematizar los estereotipos y las relaciones sociales basadas en desigualdades de género.

2. Con el objetivo de resguardar la identidad de les sujetes que han colaborado con esta investigación, se mantiene el anonimato de los barrios. Los nombres utilizados son ficticios.

3. El corpus de la tesis contó con 31 entrevistas a autoridades, coordinadores, profesionales, operadores barriales, operadoras juveniles, destinatarias e integrantes de OSC, y con 88 registros de campo.

4. El programa forma parte del Centro de Estudios Desigualdades, Sujetos e Instituciones (Cedesi - Unsam).

5. El Programa y el Centro Juvenil comenzaron a trabajar colaborativamente en el año 2015 y desde entonces, hasta el 2018 impulsaron diferentes proyectos. Agradezco profundamente a las Dras. Llobet y Medan, directoras del Cedesi y del Programa respectivamente, por invitarme a diseñar y adelantar junto con ellas la experiencia investigativa del año 2016. 
Esta experiencia, que se desarrolló durante un año, se enmarcó en un taller ya existente en el Centro, denominado proyecto de vida, y llevado a cabo de forma alternada por cuatro talleristas: una de ellas era trabajadora del Envión de La Llamita y coordinadora del mencionado taller; las otras tres, entre ellas yo, nos desempeñábamos como investigadoras del Programa de Estudios Sociales en Género, Infancia y Juventud. En el marco de esta pesquisa, produjimos 31 registros escritos de lo observado en cada taller y de las charlas informales que manteníamos con las destinatarias y les trabajadores del Centro Juvenil. Con base en dichos registros escribimos dos informes que fueron entregados y discutidos con aquelles agentes estatales.

El análisis en ambas investigaciones se centró en dos núcleos de datos: las interacciones cotidianas entre las talleristas y les agentes estatales encargades de implementar los programas, y las destinatarias y los discursos e ideas que aquelles manifestaron en el marco de las entrevistas semiestructuradas y en profundidad, así como en las diversas charlas informales.

\section{Envión: bases de un programa para la juventud excluida}

Envión es un programa de transferencias condicionadas de ingresos, destinado a personas de edades comprendidas entre 12 y 21 años, residentes en la provincia de Buenos Aires y en situación de vulnerabilidad social ${ }^{6}$. Al realizar las dos investigaciones que aquí se recuperan, los objetivos formales del programa eran integrar a les jóvenes en el sistema educativo y el mercado laboral, y ofrecerles un espacio de contención donde pudieran realizar actividades deportivas, recreativas y culturales ${ }^{7}$. Si bien el programa dependía del Ministerio de Desarrollo de la provincia de Buenos Aires, su implementación se encontraba a cargo de las áreas de niñez y adolescencia y de juventud de los municipios ${ }^{8}$. Así, las actividades de Envión solían desarrollarse en dispositivos locales destinados a jóvenes donde también se implementaban otros programas y políticas públicas para elles, y que correspondían a distintos niveles de gobierno. En San Martín, el programa Envión se implementaba en los centros juveniles del distrito, en Morón y en las casas de la juventud locales. Como lo señalan Medan, Gaitán y Llobet (2019), estos centros y casas, a diferencia de otros dispositivos de protección, como, por ejemplo, los Servicios Locales, se caracterizan por ser

\footnotetext{
6. La transferencia económica es percibida directamente por cada joven.

7. Véase https://www.gba.gob.ar/desarrollo_de_la_comunidad/asistencia/envion

8. Actual Ministerio de Desarrollo de la Comunidad.
} 
espacios organizados para que les jóvenes los transiten y habiten sin mediaciones burocráticas de turnos, y con horarios flexibles.

Al igual que otras iniciativas similares destinadas a la inclusión social de jóvenes, producto de una preocupación generacional, Envión buscaba que les destinataries lograsen construir un proyecto de vida; la incorporación al mercado de trabajo formal resulta su más acabada expresión (Gaitán, 2019; Llobet, 2009; Medan, 2013). En su diseño programático se sugería que en las sedes se trabajase sobre las capacidades subjetivas de los jóvenes de manera que puedan construir proyectos de vida propios a través de una transferencia condicionada de ingresos, además de asistencia legal, acompañamiento y oferta de espacios de formación y recreación.

En las dos implementaciones del programa estudiadas, no tener un proyecto de vida ${ }^{9}$ aparecía esbozado por les trabajadores como un riesgo que amenazaba la inclusión social de les destinataries; algo sobre lo que elles y les jóvenes debían trabajar, y que suponía un conjunto de compromisos y obligaciones. Así lo mencionó una de las trabajadoras del Envión de Morón:

Porque son pibes que vienen como más... en principio, supongo que, por las características de ellos, vienen más pensando o estando en lo inmediato, en lo cotidiano, pero como un presente muy presente, no hay ningún tipo de proyecto, es difícil detectar lo que les pasa, a veces los pibes en la cabeza es un quilombo, ya para uno a veces es difícil decir 'Bueno, me pasa esto, necesito esto'. Y si bien nosotros podemos ayudarlos en esto de la escucha, no son cosas que estén acostumbrados, entonces, para ellos también es un espacio donde se encuentran con un otro institucional, donde tienen que cumplir un determinado rol que es darle las respuestas que el profesional le pide; si pegás buena onda el pibe se va a abrir un poco más, pero todo eso es un proceso, pero a la vez, el proceso es algo que tiene que pasar, porque yo [en relación a un destinatario] estoy cobrando una beca, entonces, hacia algún lugar... (profesional, comunicación personal, 03.10.2014)

Poner a les jóvenes en una inmediatez donde la idea de un proyecto de vida se encontraba ausente constituía la justificación que Envión -y otros programas similares- utilizaba para ubicarles como personas carentes. Al esbozar la juventud como una etapa incompleta y de transición hacia un estado supuestamente pleno de adultez, el programa Envión establecía una preocupación transicional en que la

9. El concepto de proyecto de vida es una categoría paradigmática. Esta emergió alrededor de la década de 1950 y en el auge del modelo del Estado de Bienestar, asociada con las tareas definidas como propias del fin de la adolescencia: salida del hogar parental mediante la independencia económica lograda a través de un empleo o profesión, y formación de una familia propia (Llobet, 2008). 
edad aparecía, en gran parte, como la dimensión a la que invocaba para comenzar a definir a sus destinataries y sus intervenciones (Medan, 2013).

Si bien la edad constituía la primera preocupación sobre la cual desplegar acciones, esta se intersectaba en el discurso de les trabajadores de las dos sedes analizadas de manera compleja con la clase social y el género. De acuerdo con su mirada, ser joven en un barrio de sectores populares tornaba la inmediatez en algo aún más riesgoso. En sintonía con los datos presentados por Medan (2013), para les trabajadores del Envión de La Llamita y de La Estrella, esa malla de riesgos que envolvía a les destinataries por el hecho de ser jóvenes de aquel barrio atentaba contra sus posibilidades concretas de construir proyectos de vida legítimos. La carencia de un proyecto vital no solo era un fenómeno indeseable y peligroso en sí mismo, sino también producto de otras situaciones riesgosas. Por ejemplo, de necesidades básicas insatisfechas, instituciones estatales ausentes o brutalmente presentes, familias disfuncionales o ineficientes, grupos de pares inapropiados, mandatos y dinámicas barriales conflictivas (Gaitán, 2019: 293). Pero, para estes trabajadores existían diferencias según el género de les destinataries (Gaitán, 2019: 2020) ${ }^{10}$. Al igual que en otros programas con objetivos similares, ambas implementaciones del Envión reservaban la violencia, el delito y el consumo de drogas y alcohol para los varones; mientras que para las mujeres correspondían las violencias de género y una sexualidad problemática (Gaitán, 2019: 294). En sintonía con la masificación del movimiento feminista y la reactualización de sus demandas históricas, cuando se trataba de jóvenes mujeres eran la falta de autonomía y la exposición a ciertas expresiones de la violencia de género lo que las volvía vulnerables y amenazaba. Por lo tanto, el armado de un proyecto de vida se encontraba asociado al forjamiento de autonomía, así como a la desnaturalización y sustitución de comportamientos y relaciones violentas en que las jóvenes se encontrarían inmersas. Esto bajo el considerando de les trabajadores de que sus intervenciones coexistían con múltiples instituciones (estatales y no estatales) y microprácticas sancionatorias, que desplegaban instrucciones -en disputa- respecto de cómo aquellas jóvenes debían vivir sus vidas y desarrollar una feminidad apropiada. También con la reinstalación de discursos públicos (mediático y judicial) que ponían en circulación expresiones negativas de las biografías de las jóvenes pobres víctimas de violencias de género, con el objeto de culpabilizarlas de la violencia de la que eran blanco (Arduino, 2014).

Desde estas significaciones y preocupaciones, en las implementaciones locales del Envión estudiadas se forjaban propuestas de participación específicas para las destinatarias, tendientes, entre otras cuestiones, a neutralizar la violencia expresiva

10. Las interpretaciones e intervenciones de les trabajadores de ambos enviones solían montarse sobre estructuras de sexo-género binarias y cisnormadas. 
de la que son blanco (Llobet, 2015), y cuestionar y desandar sus intenciones y efectos domesticadores de sus experiencias juveniles y feminidades.

Para concluir este apartado, cabe señalar que dichas propuestas se desplegaban en el marco de una ausencia, en los ámbitos nacional y provincial, de políticas públicas integrales y líneas de acción específicas para la prevención y erradicación de las violencias contra las jóvenes mujeres.

\section{Talleres de chicas: una apuesta para gestionar las violencias de género}

Al igual que el discurso de derechos y de inclusión social, el de género guiaba parte de las prácticas de los dos equipos del Envión considerados. A pesar de que el diseño del programa no hacía referencia a la desigualdad de género, ambos equipos la habían puesto como uno de los temas prioritarios de la intervención.

En el caso del Envión La Estrella de Morón, les trabajadores habían destinado numerosos esfuerzos a cumplir con los lineamientos establecidos por el Plan $\mathrm{Mu}$ nicipal de Igualdad de Oportunidades entre Varones y Mujeres (PIO) ${ }^{11}$ : entre ellos, articulaciones con otros dispositivos del área de Género; asambleas $^{12}$ destinadas a los ejes de educación sexual integral y de aborto; y actividades en conjunto con trabajadoras del área de Salud para repasar la utilización de los métodos anticonceptivos. Por su parte, el equipo del Envión La Llamita de San Martín también consideraba que la igualdad de derechos entre les jóvenes era un tema central por atender, y por ello sostenía vínculos con dos actores estatales clave: el Centro de Atención Primaria de la Salud (CAPS) del barrio y el Programa de Estudios Sociales en Género, Infancia y Juventud de la Universidad Nacional de San Martín.

Como parte de las prioridades establecidas desde ambas implementaciones, y frente a la preocupación por el incremento de las ausencias de las destinatarias en otros espacios colectivos, en La Estrella y en La Llamita les trabajadores habían configurado propuestas de participación específicas para ellas. En el caso del primer

\footnotetext{
11. Morón no se encontró ajeno al giro subnacional en los procesos de elaboración y ejecución de políticas de igualdad de género que se produjo a la par de la emergencia de nuevas agendas globales para los derechos de las mujeres (Rodríguez-Gustá, 2010). En el año 2004 se creó la Coordinación de Políticas de Género; tres años después se transformó en Dirección de Políticas de Género y contó con un presupuesto, personal y oficinas propias (Rodríguez-Gustá, 2010). Un intento por transversalizar la política de género fue la elaboración e implementación de Planes de Igualdad de Oportunidades entre Varones y Mujeres (PIO) (2006-2008 y 2010-2014).
}

12. Entre los espacios de discusión colectivos, cada semana tenía lugar la asamblea. 
Envión, decidieron reabrir el taller de belleza y transformarlo en un grupo de mujeres que les permitiese a las destinatarias deconstruir sus identidades femeninas. Promocionado como taller de chicas, dicho espacio poco recuperó del espíritu y la dinámica del viejo taller de belleza ${ }^{13}$ y se configuró más como una propuesta para trabajar con las jóvenes cuestiones vinculadas a los estereotipos de género, la sexualidad, el placer, la autoestima, las relaciones sexo afectivas y los noviazgos. En sintonía con la observación de la coordinadora del programa en La Estrella, habían procurado "llenarlo de contenidos" (coordinadora, comunicación personal, 24.04.2014).

En el caso del Envión La Llamita, la incorporación de un espacio propio para las destinatarias surgió en el marco de la demanda del Centro Juvenil al Programa de Estudios Sociales en Género Infancia y Juventud de la (Cedesi-Unsam) ${ }^{14}$. En la planificación conjunta entre el Centro y el Programa, el espacio se pensó con una dinámica flexible que permitiese recoger los emergentes de cada encuentro e integrarlos al objetivo del proyecto: habilitar un espacio seguro y de confianza en el que las destinatarias pudiesen construir vínculos con otras mujeres a partir de problematizar los estereotipos y las relaciones sociales basadas en desigualdades de género. Como se mencionó páginas atrás, esta experiencia se desarrolló durante un año y se enmarcó en un taller ya existente en el Centro, denominado proyecto de vida ${ }^{15}$.

13. A diferencia de otros talleres que eran guiados por el equipo del Envión o por talleristas, el de belleza estaba conducido por exdestinatarias que, cobrando doble beca, habían sido reincorporadas al programa bajo la figura de operadoras juveniles. Este taller había sido propuesto por las destinatarias y buscaba construir un espacio de encuentro semanal donde aprendiesen a maquillarse y peinarse utilizando recursos gestionados por el programa. Durante el transcurso del 2013, el taller dejó de funcionar. La escasez de recursos, las desinteligencias del programa en la forma de gestionarlos y las irregularidades del pago de las becas por parte del gobierno provincial habían tornado oscilantes las asistencias de las destinatarias y de la operadora juvenil a cargo en ese momento. De acuerdo con les trabajadores, esta última había manifestado que, ante la falta de pago de la doble beca por parte del programa, su novio había empezado a cuestionar su participación en el taller.

14. En San Martín no existía un plan de igualdad de oportunidades entre varones y mujeres. De todas maneras, la Secretaría de Desarrollo Social local desplegaba acciones vinculadas a la promoción de la igualdad de derechos y oportunidades de las mujeres. Desde el año 2012, el municipio cuenta con el Sistema de Protección Integral para Mujeres que Padecen Violencia, a cargo de la Subsecretaría de Inclusión e Integración Social. Así mismo, bajo la órbita de la Secretaría de Desarrollo Social se encuentran la Dirección de Políticas de Género y la Línea Gratuita de Protección de Derechos o80o 888 5678, que atienden durante las 24 horas del día, los 365 días del año.

15. Desde el 2015, las Dras. Llobet y Medan, investigadoras del Programa de Estudios Sociales de Infancia y Juventud (Cedesi-Unsam) comenzaron a colaborar con proyectos de dos centros juveniles, entre ellos el de La Llamita. 


\section{Propuestas}

En sintonía con lo que indiqué en un trabajo previo (Gaitán, 2019:298), la construcción de ambos espacios se erigía bajo la expectativa de les trabajadores e investigadoras de que hablar de lo propio, así como escuchar lo que las otras participantes tenían para decir, permitiría a las jóvenes iniciar una introspección desnaturalizadora de sus cotidianidades. Esto les permitiría, en un escenario de múltiples constreñimientos, construir otra mirada sobre ellas mismas; modificar relacionamientos con otras mujeres, hijes, novios y familiares (Gaitán, 2019; 2020); y posicionarse desde otro lugar frente al uso del tiempo, las decisiones sobre sus cuerpos y la distribución de las tareas en el hogar (Gaitán, 2020: 293).

Por ejemplo, en una de las actividades observadas en La Estrella, las agentes estatales habían propuesto trabajar sobre estereotipos de género a partir de las ilustraciones feministas realizadas en el periodo 2014-2015 por la artista brasileña Carol Rossetti. Las ilustraciones formaban parte del Proyecto Mujer, con el cual esta diseñadora buscaba disponer mensajes positivos para las mujeres. En ese encuentro, en una ronda que trabajadoras, destinatarias y esta investigadora habíamos armado, a cada una nos correspondió una fotocopia en blanco y negro de una de aquellas ilustraciones, acompañada de frases como "El novio de Elisa decía que ella nunca podría dejarlo porque nadie más amaría una 'mujer incompleta'. Elisa No se siente incompleta desde que su amor propio y el apoyo de sus amigos, le permitieron liberarse de una relación abusiva". Cada una debía leer la suya y luego compartirla con las demás. La expectativa de las trabajadoras que coordinaban ese encuentro era que las ilustraciones y las frases funcionasen como un disparador para conversar, principalmente, sobre las relaciones entre mujeres, las parejas y los límites (Gaitán, 2019).

En el taller de La Llamita, las relaciones sexoafectivas, los noviazgos y las amistades también eran temas recurrentes porque emergía de la conversación con las jóvenes, así como por su tratamiento en el marco de actividades diseñadas para tal fin. Por ejemplo, en una oportunidad, y como parte de nuestras inquietudes como talleristas y lo que para nosotras constituía un emergente recurrente en los encuentros y en las redes sociales de las jóvenes, se trabajó sobre la dificultad y el desgano que manifestaban las destinatarias al construir amistades con otras mujeres. De la misma forma que en la actividad con las ilustraciones de Carol Rosseti en La Estrella, en La Llamita se leyó en voz alta una nota publicada pocos días antes: "La amistad entre mujeres: ser amigas, hermanas" (2016). El objetivo era reflexionar sobre los estereotipos de género puestos en juego al pensar las relaciones entre mujeres. Uno de sus primeros párrafos decía lo siguiente: 
Nos enseñaron que la amistad entre mujeres existe y que no la hay entre varones y mujeres. Pero que la amistad entre mujeres existe hasta que hay un varón en el medio. Siempre desde esa idea heterosexista y patriarcal. La rivalidad entre mujeres, la competencia, es una estructura cultural más del patriarcado. No es natural que las mujeres compitamos entre nosotras por un varón, por quien es más linda, flaca; todos los cánones de belleza que el patriarcado y capitalismo nos han impuesto. ¿A quién no le pasó? (...). ("La amistad entre mujeres: ser amigas, hermanas”, 2016)

Así, ambos espacios se encontraban orientados a la desnaturalización y apropiación reflexiva-crítica de determinadas ideas, y a la transformación de prácticas, valores y relaciones. Los dos compartían la preocupación de las jóvenes por la falta de autonomía en la toma de decisiones y la exposición a múltiples expresiones de la violencia de género, incluidas las descalificaciones públicas que condenaban moralmente sus comportamientos sexuales y las relaciones con sus cuerpos. En este sentido, resulta sugerente el siguiente pasaje; registra parte de los intercambios entre trabajadoras y jóvenes hacia el final de un encuentro destinado a elaborar el cuestionario de la encuesta sobre violencia de género, el cual aplicaríamos semanas después a vecinas del barrio La Llamita:

Angélica [tallerista] plantea que es necesario que haya una pregunta sobre salud. Sol [destinataria] asiente y dice que tiene que haber una sobre "los abortos que a veces no quieren hacer”. Claudia [tallerista] le aclara cómo es la situación del aborto actualmente en nuestro país. Sol menciona lo que Soraya [destinataria] le comentó que le ha pasado: que los médicos le habían dicho "que había estado embarazada y que había perdido el embarazo". Dice no tener muchos detalles al respecto y no continúa hablando sobre ello, pero asegura: “iNo lo iba a tener al hijo porque su mamá la mataba!” Ya llegado al final de la primera parte del cuestionario, se resuelve no incorporar toda la segunda parte sobre violencia obstétrica y, en cambio, incorporar una pregunta más general sobre la atención recibida por las mujeres en el sistema de salud. Claudia termina leyendo las preguntas del último tramo de la encuesta que son los datos sociodemográficos. Sol pregunta si cuando los padres son violentos con las hijas también es violencia de género. Claudia responde que sí y trae como ejemplo aquellas situaciones en las que las madres les dicen a sus hijas “ino te comportes como una puta!” Angélica plantea que la violencia de género "no está afuera”. (Registro de campo, 11.07.2016)

Si bien las jóvenes decidieron dejar afuera del cuestionario preguntas sobre la violencia obstétrica y en instituciones de salud, las talleristas resolvimos continuar trabajando sobre estos ejes e incorporar la temática de aborto al taller de chicas ${ }^{16}$. Esto 
se realizó a partir del trabajo durante tres encuentros y tomando como disparador el caso de Belén, una joven tucumana pobre, que en marzo del 2014 fue detenida luego de tener un aborto espontáneo en un hospital público: del hospital, con dolores abdominales fuertes, pasó al penal, esposada. En 2016, el caso se hizo público luego de que la letrada feminista Soledad Deza se convirtiera en su abogada, y organismos nacionales e internacionales de derechos humanos y los feminismos transformaran ese evento obstétrico adverso, convertido en un caso policial y luego judicial, en un hecho político (Deza, 2016). Si bien Belén era un poco mayor que las destinatarias y vivía en la otra punta del país, la atención del personal de salud aquel 21 de marzo de 2014 y el tratamiento que hizo de su caso el poder judicial se encontraban plagados de prejuicios y estereotipos de género, tal como algunas de las consultas y relaciones que las jóvenes mantenían con el sistema de salud, y que emergían en conversaciones que ellas o nosotras iniciábamos. El caso de Belén era político: se trataba de una mujer presa por abortar y permitía trabajar con las destinatarias la ciudadanía debilitada de las mujeres jóvenes de sectores populares, e identificar qué actores se constituían en garantes del derecho a interrumpir sus embarazos: quiénes nos cuidan y quiénes no. Durante esos tres encuentros, en los que se reflexionó sobre aborto, salud sexual y reproductiva, derechos de las mujeres y violencias obstétrica e institucional, se leyeron noticias periodísticas sobre el caso de Belén; se conversó con una médica generalista y directora del Centro de Atención Primaria de la Salud del barrio la Llamita, e integrante de la Red de Profesionales de la Salud por el Derecho a Decidir; y las jóvenes elaboraron dos materiales audiovisuales. Las discusiones y el trabajo realizado en el taller de chicas sobre el derecho al aborto y la criminalización de las mujeres pobres que acceden a la práctica, da cuenta de la labor reflexiva implicada en la propuesta de los talleres analizados.

\section{Respuestas}

En ambas investigaciones se vislumbró que las destinatarias experimentaban múltiples formas de violencias en sus vidas cotidianas. Era común que, en el marco

mujer que se causara o consintiera esa práctica. Aun así, se reconocían como no punibles los casos en que se encontraba en peligro la vida o salud de la mujer embarazada, o cuando el embarazo provenía de una relación sexual no consentida. A pesar de que la legislación argentina contemplaba causales en que el aborto no era punible, las mujeres en dichas circunstancias no podían -en gran medida- acceder a un aborto legal y seguro en los servicios públicos de salud. Allí, muches profesionales insistían en exigir autorización judicial para proceder con la interrupción del embarazo. Esto se debía al desconocimiento de los permisos de la ley penal, al temor a persecuciones criminales o a la objeción de conciencia. Durante la escritura de este artículo, el congreso argentino legalizó, en diciembre de 2020, la interrupción voluntaria del embarazo en las primeras 14 semanas de gestación. 
de conversaciones que manteníamos por fuera de los talleres, hablaran sobre las situaciones judiciales y penales de los varones de sus vidas, y sobre las relaciones que ellos tenían con la polícia y el delito. Como me lo comentaba Gala una tarde mientras merendábamos con su hije más pequeñe:

Gala: él [su pareja] tiene... la carátula es homicidio, robo, portación. Pero igual le hizo 9 meses y salió porque se comprobó que nada que ver. Si fue o no fue, se comprobó que no. Le hizo 9 meses, que fue un montón, pero a la vez no fue nada, de 22 años a 9 meses... Y él fue a juicio y yo tenía que presentarme como testigo. Dos días era el juicio y fue el primer día y ya le dijeron que salga, o sea, menos mal, porque si yo hubiera declarado, yo me pongo nerviosa y le hago entrar en cana ${ }^{17}$

Ana: ¿y por qué tenías que declarar vos?

Gala: porque él supuestamente el día del homicidio, digamos, él declaró que estaba en el baile conmigo y con la hermana y unas amigas, todas teníamos que declarar. Y yo le dije 'Yo me pongo nerviosa, sea o no sea verdad me pongo nerviosa, vas a quedar preso por mi culpa' [risas]. Entonces, no. Y justo él salió en septiembre y para cuando él nació [hijo menor destinataria], tuvo el juicio a los días, yo le decía que no, que no; fue el bebé [hijo menor destinataria] y Luis [hijo mayor destinataria] estaba, fuimos pero no... el primer día del juicio fue él [pareja] solo con la madre, yo no fui, y el segundo ya me dijo que no tenía que ir a declarar: un alivio. (comunicación personal, 24.11.2015)

El nerviosismo que a muchas de ellas les causaba el hecho de declarar frente a un tribunal por alguna de las causas judiciales de sus parejas o familiares, y los largos viajes a los penales a visitarlos, eran temas de conversación recurrentes. Muchas veces, cuando eran sus novios los que se encontraban alojados cumpliendo condenas, lo hacían a escondidas de su familia y con el temor de ser descubiertas. Algunas de las jóvenes, las que eran madres, también recorrían e interactuaban de forma cotidiana con otras instituciones estatales, tales como los jardines de infantes, el centro de salud del barrio y los hospitales donde hacían los controles de salud más importantes y habían tenido a su(s) hije(s). Los relatos de sus partos se configuraban en torno al recuerdo de miedos, expectativas, ansiedades y retos de familiares y médicos; y casi siempre derivaban en algún chiste para descomprimir. A veces los retos también provenían de sus parejas: en ciertas ocasiones aparecían poniendo restricciones a sus decisiones no solo respecto de sus cuerpos y las relaciones que establecían con otros pares (principalmente varones), sino también frente al uso de sus tiempos. En esas mismas conversaciones flotaban ilusiones y deseos de las jóvenes de conseguir 
algún trabajo que, aunque mal pago, les permitiera tener un tiempo solo para ellas, fuera del barrio, lejos de sus casas y del cuidado de sus hijes y hermanites. Pero, cual burbuja, esas oportunidades laborales surgían y se desvanecían con rapidez por su informalidad y precariedad -hecho que las tornaba inconstantes-, así como por la falta de apoyo y desaprobaciones de sus familiares y, a veces, de sus parejas, que en ocasiones les pedían quedarse en sus casas. Por ejemplo, Mora estaba constantemente en la búsqueda de encontrar algún empleo cuyos ingresos le sirviesen para complementar la doble beca que recibía por ser la operadora juvenil del Envión en La Estrella. Concretar esto era algo que deseaba intensamente, no solo porque entendía le permitiría tener y administrar su propio dinero como quisiera, sino porque consideraba que le posibilitaría poner un corte a las tareas domésticas, aunque solo fuera por unas horas: "salir [de su casa] y despejarme" repetía cuando tomábamos mate en la sede del Envión de La Estrella. También, disgustada, nos contaba a otras destinatarias y a mí que ese deseo solía toparse con la negativa de Darío, su novio y padre de sus dos hijas: se oponía a que ella trabajase fuera del hogar. Embarcada en continuas confrontaciones, en una oportunidad, Mora decidió separarse para "poder hacer sus cosas": "me había prohibido ir al Envión", confesó en una entrevista con otras destinatarias. Estas negociaciones, en las que cuestionaba mandatos tradicionales, podían ser más abiertas y disruptivas-separarse un tiempo-, o más silenciosas -anotarse a una capacitación sin consultarle nada-, y una vez hechas, le transmitía su resolución con cierto temor. A pesar del agotamiento que le causaban las acciones de control de Darío, su falta de apoyo para que trabajase fuera de la casa y los celos que se incrementaban durante los períodos en que él consumía sustancias psicoactivas, mantener esa relación, que Darío se hiciera cargo de ella y sus hijas en términos afectivos y económicos, era algo que Mora valoraba y le causaba orgullo. Era un logro dentro de lo que ella me planteaba como sus expectativas; estas giraban en torno a tres metas, de las cuales había conseguido cumplir dos: formar una familia y lograr vivir sola con su pareja y sus hijas en una casa. Le quedaba conseguir un trabajo pago.

Así, las destinatarias no se encontraban ajenas a los debates que se producían en sus entornos cotidianos sobre los modos apropiados de ser mujer y madre, y a veces compartían en los talleres temores y situaciones que les causaban angustias. Pero, por lo general, no lo hacían en términos introspectivos, ni con la expectativa de reflexionar en torno a la forma como sus experiencias se encontraban vinculadas a los modos genéricos restrictivos del patriarcado, como lo esperábamos las talleristas. Por ejemplo, podían despotricar contra los controles y los malos tratos de sus novios, pero aun así no se identificaban colectivamente como víctimas de violencia de género, ni iniciaban acciones comunitarias sobre dicha temática, como se esperaba desde la institución. Muchas de ellas aprovechaban los espacios para salir de sus casas y 
evadir tareas domésticas, y para construir diálogos complejos con pares mujeres. En esos diálogos podían compartir información valiosa relacionada con médicos y ayuda social, por ejemplo; también podían hacer circular chismes y bromas, con los que opinaban sobre los comportamientos maternales y las feminidades de otras jóvenes que conocían por pertenecer al barrio. La acción de chismear y bromear sobre esas otras mujeres ofrecía un espacio central de circulación de comentarios, datos y anécdotas que permitía a las jóvenes reforzar posiciones morales frente a nosotras (Fassano, 2008).

A manera de ejemplo de lo anterior, cuando se discutió la temática del aborto en La Estrella, lejos de arribarse a un consenso respecto de cómo dicho derecho se encontraba restringido para las mujeres, varias de las destinatarias respondieron mencionando y condenando a sus pares del barrio que habían decidido interrumpir sus embarazos. Algo similar, pero con otro tema, había ocurrido en La Llamita cuando, luego de hacer una encuesta sobre violencia de género en el barrio, se pretendió profundizar en una de las preguntas: la circulación de las mujeres en el espacio público.

Verónica [tallerista] plantea que, en algunas oportunidades en el espacio, ellas han utilizado el término "callejera" o "callejear", les pregunta qué significan esos términos y en un principio todas se quedan calladas. Las coordinadoras insistimos con la pregunta. Ramona responde con cara de como si no fuera nada y nos dice que significa hacer cosas por la calle, estar por la calle, nada más. Enseguida Selva [su tía, apenas unos años mayor que ella] se ríe y le dice que hay una diferencia entre su andar por la calle y el de ella. Resalta que ahora solo sale con su marido, o va a lo de la mamá con la bebé, no como Ramona y Carolina [hermana de Selva] que sí callejean. Ramona y Carolina también ríen y le responden “icallate, sucia!”. Entre ellas suelen decirse "sucia” como broma. Rocío [otra destinataria] en cambio, responde por lo bajo y en tono más serio y con un dejo irónico “depende de lo que hagas, está bien o mal” [estar en la calle]. Cuando Verónica le pregunta qué quiere decir con eso, Rocío no lo amplía y permanece callada. (Registro de campo, o8.08.2016)

Luego de ese intercambio, Ramona y Carolina dejaron la sala y la actividad. Según lo que comentaron las jóvenes, otra amiga que no participaba del espacio había pasado a buscarlas para ir a pasear. Al partir, y nuevamente con una sonrisa, Selva nos dijo que en verdad se iban a callejear, más ahora que su prima ya no estaba de novia. $\mathrm{Al}$ quedar menos mujeres en el espacio, Selva y las talleristas continuaron conversando sobre el tema, mientras las otras destinatarias optaron por guardar silencio.

Que se fueran antes de los talleres o que se quedaran en silencio frente a una propuesta de trabajo formaba parte de los modos en que muchas de las destinatarias participaban de las propuestas diseñadas para ellas. Como se indicó en un trabajo 
previo (Gaitán; Llobet; Medan, 2015), si bien el silencio puede ser interpretado como una señal de resistencia activa con el objeto de no responder verbalmente a la interpelación (MacLure; Holmes; Jones; MacRae, 2010), también puede ser entendido como una manifestación no de una resistencia a la interpelación a hablar, sino más bien, al análisis de les que intervienen -en este caso, las talleristas-; puede constituir un límite a la interpretación y a las posibilidades de intervención (Gaitán et al., 2015). Ese silencio circulaba en las propuestas de participación: un silencio que escondía algo y cuya presencia inquietaba e incomodaba a las talleristas, quienes solíamos arriesgar interpretaciones al respecto. Así lo indicó en una mañana una de las profesionales que había participado en la planificación del taller de belleza en La Estrella, y que estaba a cargo de otros talleres (como Jóvenes y Memoria ${ }^{18}$ ):

Todo el tema de la violencia, si bien los convoca, porque ellos hay una identificación... en realidad hay una vivencia, yo ahí me estoy replanteado si ellos realmente después cuando vienen a la Casa de la Juventud tienen ganas de hacer eso o de encontrarse con pares y boludear y hablar de otro tipo de cosas. Yo, en Jóvenes y Memoria, siempre como que estoy coordinando y proponiendo, es un espacio para mí más de investigación y de denuncia sobre los derechos humanos, es un lugar donde está bueno poder hablar de esas cosas. El tema es que yo veo que se arma debate, pero por ahí con algunos no, porque quieren estar, pero no tienen ganas de estar hablando de cosas serias (...) por ahí hacer, no sé, el insight de pensar lo que te pasa, lo que te duele, la injusticia y todo eso, es algo medio pesado. (Profesional, comunicación personal, 03.10.2014)

En este mismo sentido, luego de cinco meses de realizar el taller en La Llamita, y en el marco de una reunión de evaluación de la propuesta, las talleristas escribimos lo siguiente:

Las chicas dejaron de ir al taller cuando les preguntamos o les insistimos que entren al espacio. Tenemos que hacer actividades más formales y estructuradas, pero tratando de bajar menos línea ${ }^{19}$, para que hablen más, porque últimamente, como parte de hacer una estrategia más horizontal, contamos mucho sobre nuestras propias vivencias y con eso estamos bajando una línea que quizás, o probablemente, choque con sus propios valores y formas. (Registro de campo, 12.09.2016)

18. Dicho programa se encuentra coordinado por la Comisión Provincial por la Memoria de la Provincia de Buenos Aires. Su objetivo es lograr que les jóvenes investiguen, narren y se apropien de las experiencias del pasado reciente de manera colectiva.

19. Forma coloquial en Argentina de expresar que se instala una idea o pensamiento determinado en otra(s) persona(s). 
La existencia de esos silencios de lo no hablado emergía de la complejidad de las vidas cotidianas de las destinatarias del Envión de La Estrella y La Llamita. Ellas se encontraban insertas en un entramado complejo de relaciones sociales, de contingencias y de experiencias vividas. Sus historias sobre las relaciones con sus hijes, los varones y las otras mujeres eran ambiguas y enmarañadas. Comprenderlas en su complejidad requería una escucha atenta, minuciosa y empática, de un ejercicio constante por atender al modo en que las relaciones de género, cómo toda relación social, al ser multidimensionales, resultaban irreductibles a un único y universal set de prioridades acordadas. Las talleristas reconocíamos esto y hacíamos esfuerzos por mantenernos atentas y críticas respecto de cómo, algunas de nuestras "expectativas de clase media", se colaban en nuestras prácticas. De todos modos, la pretensión de hacerlo no significaba que lo lográramos siempre.

\section{Reflexiones abiertas}

En el marco de ambas investigaciones se vislumbró que las destinatarias del programa Envión experimentaban diferentes formas de violencias en el marco de sus vidas cotidianas en los barrios. Allí, donde la precariedad desbordaba las situaciones de trabajo y se encontraba extendida a otras esferas de la vida-condiciones habitacionales, alimentarias o sanitarias-(Fernández-Álvarez; Pacífico; Señorans, 2020), aquellas jóvenes, ubicadas en posiciones asimétricas respecto de sus pares varones y otres adultes de su grupo de parentesco y del contexto barrial, e incluso de trabajadores estatales e investigadoras, convivían y experimentaban diferentes formas de violencia, las cuales organizaban sus experiencias y relaciones. Sus experiencias de exclusión y vulnerabilidad se encontraban asociadas a expresiones de violencia social en su faceta generacional, de género e institucional. Si bien los programas sociales como el estudiado trataban de comprender estas asociaciones en aras de proponer soluciones que garantizasen el derecho de las jóvenes a vivir vidas libres de violencias y autónomas, encontraban obstáculos al no comprender en su totalidad cómo las desigualdades culturales, espaciales y económicas se intersectaban e interactuaban entre sí. Esto se evidenciaba en el relativo conocimiento de las formas en que las violencias circulaban entre las destinatarias no solo desarmando relaciones, sino también construyendo universos sociales (Das, 2007b).

Los límites en la interpretación respecto a la manera en que las formas de violencia y precariedad atravesaban y atraviesan la vida cotidiana de las jóvenes de sectores populares, al tiempo que organizan sus relaciones y sensibilidades en los barrios, no se han producido solo en el ámbito de las políticas de inclusión social 
y de las militancias feministas; también se han presentado en el de las ciencias sociales. En este último caso, dicho límite se relaciona con la escasa articulación analítica entre los procesos de violencia social y la regulación de la población juvenil en su intersección con otros clivajes identitarios, tales como la clase y el género (Creenshaw, 1989). Solo algunos trabajos recientes (Llobet, 2015) han comenzado a atender esta situación.

Habida cuenta del señalamiento hecho desde la antropología sobre la cotidianidad e inestabilidad de la violencia (Das, 2007a; 2008; Garriga-Zucal; Noel, 2010; Han, 2012), y de que tal inestabilidad, producto de la relacionalidad de las experiencias de los sujetos, es crucial para comprender cómo la realidad de la violencia incluye su virtualidad y su potencial para hacer y deshacer mundos sociales (Das; Kleinman; Mamphela; Reynolds, 200o), parece entonces significativo enfatizar en los siguientes interrogantes: ¿El miedo y las confrontaciones que impregnan los relatos y recuerdos de experiencias centrales en las vidas de las jóvenes pueden desarmar y crear de forma simultánea nuevas formas de relacionamientos y prácticas? ¿De qué manera esas múltiples violencias inciden en la configuración de las construcciones de autonomía? ¿Exhiben las violencias un costado productivo? ¿Comparten territorio con los afectos? ¿Cuáles son los efectos regulatorios de las formas de violencia y la precariedad que estas jóvenes experimentan como parte de ser jóvenes mujeres en un barrio empobrecido? ¿Las experiencias y las historias de violencia les permiten construir posiciones en un marco de múltiples constreñimientos? Estas preguntas resultan significativas para un campo en conformación en la Argentina respecto de las formas de circulación y regulación de las violencias entre jóvenes, que hasta el momento se ha centrado principalmente en la relación de los varones con el delito y las fuerzas policiales (Cozzi, 2015; Rodríguez-Alzueta, 2016). Para responderlas será necesaria una aproximación a las violencias que reclame no solo atender las estructuras económicas e institucionales, locales y globales que la causan, como lo hacen lecturas más estructuralistas del fenómeno (Monárrez-Fragoso, 2009; Segato, 2003), sino también una mirada etnográfica que preste atención a los modos en que las diversas formas de violencia traman los márgenes urbanos en la cotidianidad (Das 2007b; Ortega, 2007); en palabras de Jimeno (2019), apostar a un vaivén constante entre las dimensiones estructurales de la violencia y su vivencia individual. Propuesta que ya hiciera la antropología sobre el sufrimiento social, ligada a la búsqueda de nuevas formas de pensar e interpretar las complejas relaciones entre actos de violencia, significación, representación, hegemonía o resistencia (Ferrándiz-Martín; Feixa-Pampols, 2004: 160). En consonancia con investigaciones locales (Castilla, 202O; Franco-Patiño; Llobet, 2020; Llobet, 2020), el asunto radica en desarrollar una aproximación que revele la manera como las violencias, los cuidados, las insti- 
tuciones estatales y la precariedad se articulan en relaciones íntimas, compromisos y expectativas de las jóvenes de sectores populares (Han, 2012).

\section{Referencias}

Arduino, Ileana (2014). Melina Romero. La Mala Víctima. Revista Anfibia. Recuperado de http://revistaanfibia.com/ensayo/la-mala-victima/

Castilla, María Victoria (2020). Violencias, amor y cuidados maternos en mujeres pobres de Buenos Aires. Etnografias Contemporáneas, 6(10), 132-153.

Cerletti, Laura; Gessaghi, Victoria (2012). Clases sociales, trabajo de campo y desigualdad, discusiones a partir del enfoque etnográfico. Publicar en Antropología y Ciencias Sociales, $13,31-48$.

Cornwall, Andrea (2008). Unpacking 'Participation': Models, Meanings and Practices. Community Development Journal, 43(3), 269-283.

Cozzi, Eugenia (2015). De juntas, clanes y broncas: Regulaciones de la violencia altamente lesiva entre jóvenes de sectores populares en dos barrios de la ciudad de Santa Fe. Delito y Sociedad, 1(39), 72-102. Recuperado de https://ri.conicet.gov.ar/handle/11336/52816

Cozzi, Eugenia (24 de marzo de 2019). No toda la violencia que se ve en los barrios está ligada al narcotráfico. La Capital. Recuperado de https://www.lacapital.com.ar/policiales/notoda-la-violencia-que-se-ve-los-barrios-esta-ligada-al-narcotrafico-n1748895.html

Crenshaw, Kimberlé (1989). Demarginalizing the Intersection of Race and Sex: A Black Feminist Critique of Antidiscrimination Doctrine, Feminist Theory and Antiracist Politics. University of Chicago Legal Forum, 1989(1), 138-67.

Das, Veena (2007a). Life and Words: Violence and the Descent into the Ordinary. Berkeley: University of California Press.

Das, Veena (2007b). La antropología del dolor. En Veena Das: sujetos de dolor, agentes de dignidad (pp. 69-94), editado por Francisco Ortega. Bogotá: Universidad Nacional de Colombia.

Das, Veena (2008). Violence, Gender, and Subjectivity. The Annual Review of Anthropology, $37,283-299$.

Das, Veena; Kleinman, Arthur; Ramphele, Mamphela; Reynolds, Pamela (Eds.) (2000). Violence and Subjectivity. Berkeley: University of California Press.

Deza, Soledad (2016). Libertad para Belén. Grito Nacional. Buenos Aires: Cienflores. 
Fassano, Patricia (2008). El chisme: una práctica que performatiza la sociabilidad del barrio. Trabajo presentado en el IX Congreso Argentino de Antropología Social, Facultad de Humanidades y Ciencias Sociales, Universidad Nacional de Misiones, Posadas, Argentina.

Fernández-Álvarez, María Inés; Pacífico, Florencia; Señorans, Dolores (2020). Parar la olla durante la pandemia. Revista Anfibia. Recuperado de http://revistaanfibia.com/ensayo/ parar-la-olla-durante-la-pandemia/

Ferrándiz-Martín, Francisco; Feixa-Pampols, Carles (2004). Una mirada antropológica sobre las violencias. Alteridades, 14(27), 159-174. Recuperado de https://www.redalyc.org/ pdf/747/74702710.pdf

Franco-Patiño, Sandra; Llobet, Valeria (2019). Los Centros de Desarrollo Infantil y los procesos de institucionalización del cuidado de la infancia en la provincia de Buenos Aires. Dinámicas territoriales de género. En Marchas y contramarchas en las políticas locales de género (pp. 59-86), editado por Ana Laura Rodríguez-Gustá. Buenos Aires: Clacso. Recuperado de http://biblioteca.clacso.edu.ar/clacso/gt/20200227055551/Marchas-y-Contramarchas.pdf

Gaitán, Ana Cecilia (noviembre, 2017). Juventud y maternidad en el barrio. Etnografia de las negociaciones de sentidos y prácticas en la implementación de políticas sociales en el conurbano bonaerense [Tesis de doctorado]. Universidad de Buenos Aires, Buenos Aires, Argentina.

Gaitán, Ana Cecilia (2019). Construir “otra mirada”. Tensiones en la participación de jóvenes mujeres en una política social en el Área Metropolitana de Buenos Aires. En iAquílosjóvenes! Frente a las crisis (pp. 284-310), coordinado por Gerardo Gutiérrez Cham y Olaf Kaltmeier. Guadalajara: Universidad de Guadalajara.

Gaitán, Ana Cecilia (2020). Juntas, hablar de lo propio y escuchar lo de otras. Efectos imprevistos y tensiones en una propuesta estatal de participación juvenil centrada en la autonomía femenina. En Infancias y juventudes a 30 años de la Convención sobre los Derechos del Niño. Políticas, normativas y prácticas en tensión (pp. 277-308), compilado por Paula Isacovich y Julieta Grinberg. José C. Paz: Edunpaz.

Gaitán, Ana Cecilia; Llobet, Valeria; Medan, Marina (2015). “ ¿Alguien por casualidad quiere decir algo?" Reflexiones sobre las interpretaciones de los silencios en programas de inclusión para jóvenes. Servicios Sociales y Política Social, 107, 101-114.

Garriga-Zucal, José; Noel, Gabriel (2010). Notas para una definición antropológica de la violencia: un debate en curso. Publicar, 8(9), 97-121.

Guber, Rosana (2004). El salvaje metropolitano Reconstrucción del conocimiento social en el trabajo de campo. Buenos Aires: Paidós.

Han, Clara (2012). Life in Debt: Times of Care and Violence in Neoliberal Chile. Berkeley: University of California Press. 
Jimeno, Myriam (2019). Cultura y violencia: hacia una ética social del reconocimiento. Bogotá: Universidad Nacional de Colombia.

La amistad entre mujeres: ser amigas, hermanas (2016). Marcha: una mirada populary feminista de la Argentina y el mundo. Recuperado de https://www.marcha.org.ar/la-amistad-entremujeres-ser-amigas-hermanas/

Llobet, Valeria (2008). Las políticas sociales para la infancia, la psicología y el problema del reconocimiento. Investigaciones en Psicología. Revista del Instituto de Investigaciones de la Facultad de Psicología, 14(2) 73-94. Recuperado de https://www.aacademica.org/valeria. llobet/74.pdf

Llobet, Valeria (2009). Género y políticas sociales para la infancia y la adolescencia: ¿una relación ausente? En Política Social en América Latina y Género: Configuraciones-Reconfiguraciones en la participación de las mujeres (pp.173-194), compilado por Susana Ortale y Rocío Enríquez. Buenos Aires: Biblos.

Llobet, Valeria (2015). Políticas y violencias en clave generacional en Argentina. En Juvenicidio: Ayotzinapa y las vidas precarias en América Latina y España (pp. 215-224), coordinado por José Manuel Valenzuela Arce. Tijuana: Colegio de la Frontera Norte.

Llobet, Valeria (2020). El encierro de los niños y la distribución desigual de la precariedad. Sociedad e Infancias, 4, 283-284. Recuperado de https://revistas.ucm.es/index.php/SOCI/ article/view/69632

Llobet, Valeria; Gaitán, Ana Cecilia; Medan, Marina; Magistris, Gabriela (2013). Este espacio es para que ustedes hablen. La legitimación de la intervención en los programas sociales. En Sentidos de la exclusión social. Beneficiarios, necesidadesy prácticas en políticas sociales para la inclusión de niñosy jóvenes (pp.129-159), coordinado por Valeria Llobet. Buenos Aires: Biblos.

MacLure, Maggie; Holmes, Rachel; Jones, Liz; MacRae, Christina (2010). Silence as Resistance to Analysis: Or, on Not Opening Ones Mouth Properly. Qualitive Inquiry, 16(6), 492-50o.

Masson, Laura (2020). Introducción del Dossier La violencia perturbadora. Mujeres y uso de la fuerza. Etnografias Revista del Centro de Estudios de Antropología, 6(10), 121-131. Recuperado de https://revistasacademicas.unsam.edu.ar/index.php/etnocontemp/article/view/524

Medan, Marina (2013). El gobierno de la "juventud en riesgo" y los programas de prevención social del delito en el AMBA: entre la seguridad y la inclusión [Tesis de doctorado]. Universidad de Buenos Aires, Buenos Aires, Argentina.

Medan, Marina; Gaitán, Ana Cecilia; Llobet, Valeria (2019). El Estado local y el territorio. Aspiraciones de cercanía y transformaciones en la institucionalidad de los sistemas de protección y promoción de niños y jóvenes. En De la desjudicialización a la refundación de los derechos. Transformaciones en las disputas por los derechos de los niños y las niñas (2005- 
2015) (pp. 315-352), coordinado por Valeria Llobet y Carla Villalta. Buenos Aires: Teseo. Recuperado de https://www.teseopress.com/desjudicializacion/

Monárrez-Fragoso, Julia (2009). Tramas de una injusticia. Feminicidio sexual sistémico en Ciudad Juárez. Tijuana: El Colegio de la Frontera Norte.

Ortega, Francisco (2007). Rehabitar la cotidianidad. En Veena Das: Sujetos del dolor, agentes de dignidad. (pp.15-69), editado por Francisco Ortega. Bogotá: Universidad Nacional de Colombia.

Rodríguez-Alzueta, Esteban (Comp.) (2016). Hacer Bardo. Provocaciones, resistenciasy derivas de jóvenes urbanos. La Plata: Malisia.

Rodríguez-Gustá, Ana Laura (2010). ¿Qué es la gestión transversal territorial de género? Una comparación de Moróny de Montevideo. Trabajo presentado en V Congreso Latinoamericano de Ciencia Política, Asociación Latinoamericana de Ciencia Política, Buenos Aires, Argentina.

Segato, Rita (2003). Las estructuras elementales de la violencia. Buenos Aires: Unqui.

Sirvent, María Teresa; Rigal, Luis (2014). La investigación acción participativa como un modo de hacer ciencia de lo social. Revista Decisión, 38, 32-37. Recuperado de https://www.crefal. org/decisio/images/pdf/decisio-48-49/decisio-48-49-arto5.pdf 